\title{
Colorectal Carcinoma in Children and Adolescents in Low Middle Income Countries: Single Center Experience
}

\author{
Wael Zekri $^{1,2}$, Soha Ahmed ${ }^{3}$, Ahmed Elhemaly ${ }^{1,2}$, Gehad Ahmed ${ }^{4,5}$, Eman Zaki ${ }^{6,7}$, \\ Naglaa Elkinaee $^{8,9}$, Amr Elnashar ${ }^{10}$, Marwan Adly ${ }^{10}$, Alaa Younes ${ }^{4,5}$, Asmaa Hamoda ${ }^{1,2, *}$ \\ ${ }^{1}$ Department of Pediatric Oncology, Children Cancer Hospital Egypt, Cairo, Egypt \\ ${ }^{2}$ Department of Pediatric Oncology, National Cancer Institute, Cairo University, Cairo, Egypt \\ ${ }^{3}$ Department of Clinical Oncology, Aswan University, Aswan, Egypt \\ ${ }^{4}$ Department of Surgery, Helwan University, Cairo, Egypt \\ ${ }^{5}$ Department of Surgery, Children Cancer Hospital Egypt, Cairo, Egypt \\ ${ }^{6}$ Department of Radio-diagnosis, Children Cancer Hospital Egypt, Cairo, Egypt \\ ${ }^{7}$ Department of Radio-diagnosis, National Cancer Institute, Cairo University, Cairo, Egypt \\ ${ }^{8}$ Department of Pathology, National Cancer Institute, Cairo University, Cairo, Egypt \\ ${ }^{9}$ Department of Pathology, Children Cancer Hospital Egypt, Cairo, Egypt \\ ${ }^{10}$ Department of Clinical Research, Children Cancer Hospital Egypt, Cairo, Egypt
}

\section{Email address:}

asmaahamoda82@hotmail.com (A. Hamoda),asmaa.hamoda@57357.org (A. Hamoda)

${ }^{*}$ Corresponding author

\section{To cite this article:}

Wael Zekri, Soha Ahmed, Ahmed Elhemaly, Gehad Ahmed, Eman Zaki, Naglaa Elkinaee, Amr Elnashar, Marwan Adly, Alaa Younes, Asmaa Hamoda. Colorectal Carcinoma in Children and Adolescents in Low Middle Income Countries: Single Center Experience. Cancer Research Journal. Vol. 9, No. 1, 2021, pp. 8-13. doi: 10.11648/j.crj.20210901.12

Received: December 18, 2020; Accepted: January 8, 2021; Published: January 22, 2021

\begin{abstract}
Colorectal carcinoma (CRC) is rare in children with extremely poor prognosis. Proper management is obscure because of deficient data. The aim of the current study is to assess the clinical characteristics and to determine the different prognostic factors leading to dismal outcome. It is a retrospective study included all CRC patients, below 18 years, treated in Children's Cancer Hospital of Egypt (CCHE) between 2007 and 2016. Demographic data, clinical characteristics, diagnostics, histological subtypes, disease stage, treatment methods, and survival outcome were collected. The result showed that, 15 patients below 18 years. All had unfavorable histopathology (mucinous adenocarcinoma) and 10 cases had metastatic disease. Initial surgical resection was done in $8 / 15$ cases, all patients received neo adjuvant/adjuvant chemotherapy. Four patients had rectal adenocarcinoma and were treated with chemo-radiotherapy while 11 had colonic adenocarcinoma. Ten patients had progression or relapse, while 12 died at the end of follow up period; 3-years Overall Survival (OS) and Event Free Survival (EFS) were $17.8 \%$ and $16.5 \%$ respectively, the only three surviving patients were of lower stage disease. In Conclusions, Clinical presentation of CRC in pediatrics is similar to adults but delayed diagnosis and advanced stages contribute to poor outcome which is due to the absence of familiarity to CRC. The high frequency of mucinous adenocarcinoma may explain the poor outcome. Surgery remains the mainstay of treatment as in adults. Preoperative radiotherapy for rectal adenocarcinoma, offers better prognosis. Chemotherapy plays a role in the metastatic disease and can downstage the primary tumor for better local control.
\end{abstract}

Keywords: Colon, Rectal, Adenocarcinoma, Childhood, Adulthood

\section{Introduction}

Colorectal carcinoma (CRC) is the third most common cancer in adults after lung cancer and breast cancer, its incidence peaks at age 55-60 years. The Surveillance, Epidemiology, and End Results (SEER) program accounted 
only about 80 new cases per year in pediatric age in the United States (out of nearly 150,000 cases of all ages), with an annual incidence of approximately one case per million individuals [1-3].

Most of reported cases are in adolescents, while incidence in the pre-pubertal cases is much lower. Because CRC is rare in children and adolescents and due to limitation of information availability on the clinical features, onset of disease, prognostic factors of CRC which hinder the ability for proper analysis for the reasons of lower survival in children and adolescents in comparison to adults [4].

The worse prognosis may be due to delayed diagnosis as it is frequently misinterpreted or missed in the differential diagnosis of abdominal pain, weight loss, and anemia. Also, advanced clinical stage at diagnosis and higher incidence of unfavorable histopathological types (high-grade, poorly differentiated subtypes) also play a role in the dismal outcome for those cases [5]. As pediatric oncologists we don't have much of experience in management of these cases that is why the treatment strategy is generally mimic to the adult experiences. Surgical management and long-term follow-up of this group of patients are still obscure because of lack of data.

\section{Aim of the Work}

In this retrospective study tried to contribute to the scanty available data in the literature about the clinical findings, investigation, multimodality treatment and outcome of pediatric CRC patients below 18 years.

\section{Patients and Methodology}

Fifteen patients with pediatric CRC under the age of 18 years were treated at the Children's Cancer Hospital Egypt (CCHE) from July 2007 to July 2016. Collecting complete data was available for all patients as regards clinical features, histolopathology, treatment modalities and outcome (which were extracted from the patients' file, office, surgeon's and pathologist's reports) and was reviewed for this analysis. All patient's guardians had approved management of their data by written consent according to the ethical rules applied in CCHE. At diagnosis, abdominal computerized tomography (CT) and chest CT scan were done for proper staging, and whole-body bone scan was done initially for all patients to exclude bone metastases. For cases of rectal adenocarcinoma, MRI pelvis was done. Colonoscopy was carried to evaluate the primary tumor site, size and to take tissue biopsy for pathological diagnosis and proper TNM staging. Tumor stage was assessed according to the modified Dukes classification6 and the American Joint Committee of Cancer (AJCC) Staging System [6].

Patients were treated using multi-modality approaches including surgery, chemotherapy and, in rectal carcinoma cases, radiotherapy and chemotherapy $+/$ - surgery were used based on the ongoing protocols at the time of diagnosis [7]. Event-free survival (EFS) and overall survival (OS) were estimated according to the Kaplan- Meier method [8]. Patients were evaluated from the result of histolopathological diagnosis till last follow-up, disease progression, or relapse for progression free survival (PFS), and till any event (disease progression/relapse, second neoplasms) for EFS, and up to death for OS.

\section{Results}

This study retrospectively analyzed 15 patients diagnosed with colorectal carcinoma in the period between July 2007 till July 2016 at the CCHE and followed up till December 2018. Five cases were males and 10 cases were females, with male: female Ratio 1:2, Median age was 15 years (Range: 918). Those cases are fairly distributed all over Egypt; Giza/ Cairo (6cases), Upper Egypt (5 cases) and Lower Egypt (4 cases). Three cases seem to have cancer predisposition syndrome; 2 cases had (Adenomatous Familial polyposis) and café au lait patches (NF1) versus possibility of constitutional bi-allelic mismatch repair syndrome "CBMMR" and one case had café au lait patches only without polyposis coli may be NF1 syndrome versus CBMMR, the three patients with café au lait patches doses not have other clinical stigmata of NF1.

The main presenting symptoms were nausea and vomiting in 7 cases $(47 \%)$, Abdominal colic in 12 cases $(80 \%)$, intestinal obstruction in 8 cases $(53 \%)$, weight loss in 13 cases $(86 \%)$ and rectal bleeding in 8 cases $(53 \%)$. Most of the cases presented lately with an average duration of 6 months between the appearances of symptoms till diagnosis.

Nine patients $(60 \%)$ presented with hemoglobin level below $10 \mathrm{~g} / \mathrm{dl}$. Eleven patients out of $15(74 \%)$ were diagnosed as colon adenocarcinoma, while 4 cases $(26 \%)$ were diagnosed as rectal adenocarcinoma from which in 2 cases the disease extended down till the anal canal. Colonic carcinoma was distributed along the colon as follows: ascending colon in 2 cases $(13.3 \%)$, descending colon in 6 cases $(40 \%)$ and sigmoid colon in 3 cases $(20 \%)$.

Ten cases were metastatic at presentation; one case $(10 \%)$ had rectal carcinoma, while 9 cases $(90 \%)$ had colonic carcinoma. Twelve cases underwent surgical resection of the tumor from which 6 cases did upfront surgical resection. While 3 cases did just a biopsy and the remaining 6 cases did surgery post Neoadjuvant chemotherapy or radiotherapy or both, four out of the 6 cases who did upfront surgical resection achieved gross total resection (3/4 achieved negative surgical margin), while 2 cases did incomplete resection with gross residual.

Six cases did delay surgical resection after Neoadjuvant chemotherapy (2-4 cycles); 4/6 cases did gross total resection; 3 of them with positive surgical margin, while one case only had margin negativity however two cases did delay incomplete resection with gross residual.

The pathological analysis of all cases revealed mucinous adenocarcinoma, signet ring type in all cases distributed between Grade 2 ( 8 cases) \& Grade 3 ( 7 cases). There were 6 cases with ascetic fluid positivity and 3 cases with peritoneal 
deposits positivity.

The metastatic sites were the ovary in 3 cases $(20 \%)$, chest in 2 Cases $(13 \%)$, peritoneum and omentum in 6 cases (40\%), bone in 3 cases $(20 \%)$, liver in 2 cases $(13.3 \%)$, cervical and mediastinal lymph nodes in one case $(6.6 \%)$.

Five cases were non metastatic $(33.3 \%), 4$ of them had regional lymph node involvement (stage III), while one case had no lymph node involvement (stage IIA).

Regarding the tumor size; T1 was present in 1 case $(6.6 \%)$, $\mathrm{T} 2$ in 2 cases $(13.3 \%)$, T3 in 7 cases $(46.6 \%)$, T4 in 5 cases (33.3\%), 2 cases were T4a and 3 cases were $\mathrm{T} 4 \mathrm{~b}$.

Metastasis in 2-3 regional lymph nodes was found in one case $(\mathrm{N} 1 \mathrm{~b})$, one case $(6.6 \%)$ was extending to the sub serosa and mesentery (N1c), metastasis in 4 or more regional lymph nodes (N2) was found in 10 cases $(66.6 \%)$, from which 7 cases were having metastases in 7 or more regional lymph nodes. Lymph nodes status couldn't be assessed in 2 cases. The staging of the 15 cases was done according to classification and the American Joint Committee on Cancer (AJCC) Staging System. 7Stage IIA in (1case), III B in (2 cases), III $\mathrm{C}$ in (2 cases), IV A in (2 cases) and IV B in (8 cases).

Neoadjuvant chemotherapy regimen were either FOLFOX, FOLFIRI or FOLFOXIRI without standardization as there were no fixed protocols in management of the cases and decision was mainly dependent on the primary physician. Evaluation was done mostly after the third course to go either for delayed surgical resection or to complete another 3 courses of FOLFOX if there is a response to Neoadjuvant treatment or to shift to the salvage treatment FOLFIRI7 if there is progression.

Five cases of colonic adenocarcinoma received Neoadjuvant chemotherapy; 3 cases received FOLFOX (4), one case received FOLFIRI and another case received FOLFOXIRI. Tumor regression occurred in 2 cases after three cycles of chemotherapy and they underwent surgery at that time; both cases continued chemotherapy for a total of 6 cycles. The other 3 cases progressed after 2-3 cycles of chemotherapy and died from the disease. Radiotherapy was not given in any of the colonic adenocarcinoma cases.

In general, the toxicity was tolerable for FOLFOX (4) with nausea, vomiting and abdominal colic as the main toxicity. FOLFIRI was severely toxic with profound neutropenia, GIT toxicity and cardio toxicity. The case which received FOLFOXIRI died from ventricular fibrillation and cardiac arrest after the 6th course of chemotherapy.

Four cases with rectal adeno-carcinoma started by Neoadjuvant chemo and radiotherapy (radiotherapy followed by chemotherapy in 3 cases while the remaining case started by chemotherapy followed by delayed radiotherapy after the 6th cycle of FOLFOX, radiotherapy was given with concomitant oral 5 Fluorouracil (Xeloda) in 2 cases or FOLFOX (4) in the other 2 cases. The dose of the preoperative radiotherapy was 5040 cGy $/ 180 / \mathrm{fr}$ over 5 weeks. The toxicity was accepted in all cases.

After ending radiotherapy, the rectal carcinoma patients continued till a total of 6 courses of 5 Fluorouracil. Three out of the 4 cases received postoperative chemotherapy, due to either positivity of the margin or lymph node, in the form of either FOLFIRI (1 case) or FOLFOXIRI ( 2 cases). The toxicity of chemotherapy was tolerable within the cases of rectal adenocarcinoma, with mild myelo-suppression and GIT symptoms and there was no toxicity related mortalities.

Surgery was attempted after ending Neoadjuvant chemo/radiotherapy where 3 cases achieved gross total resection with positive microscopic margin.

Twelve patients died and 3 were alive in CR ( 2 cases of rectal adenocarcinoma and one case of colon adenocarcinoma). By analyzing the dead patients we found that ten patients had tumor progression or relapse and died from their disease $(8$ patients of colon carcinoma and 2 patients of rectal carcinoma), while the 2 other cases of colon cancer died from another causes; one case from chemotherapy toxicity and the other from an appearance of brain tumor as a second primary malignancy which was believed to be high grade glioma and this patient was one of the three patients who were suspected to be CBMMR syndrome.

The 3-year overall survival (OS) of the cohort was $17.8 \%$ $(\mathrm{SE}+11.1)$, Figure 1, and 3-year event free Survival (EFS) $16.5 \%$ (SE + 13.5) "Figure 2" with median survival 15.7 months. (For patients' demographics, staging, therapeutic approach and survival outcome refer to, Table 1.

Table 1. Demographic data, staging, therapeutic approach and survival outcome of the study patients $(n=15)$.

\begin{tabular}{|c|c|c|c|c|c|c|}
\hline Serial & AGE (years) & Gender & Site & $\mathbf{T}$ & $\mathbf{N}$ & $\mathbf{M}$ \\
\hline 1 & 14 & $\mathrm{M}$ & colon & T3 & No & M0 \\
\hline 2 & 18 & $\mathrm{~F}$ & colon & $\mathrm{T} 4 \mathrm{~b}$ & $\mathrm{~N} 1 \mathrm{C}$ & M1a (ovary) \\
\hline 3 & 12 & M & colon & $\mathrm{T} 3$ & $\mathrm{~N} 2 \mathrm{~b}$ & M1b (chest, peritoneal, bone) \\
\hline 4 & 16 & M & colon & $\mathrm{T} 4 \mathrm{a}$ & NX & M1b (peritoneal, hepatic) \\
\hline 5 & 12 & $\mathrm{~F}$ & colon & $\mathrm{T} 3$ & N2 & M1b (bone, cervical and mediastinal lymph nodes) \\
\hline 6 & 17 & $\mathrm{~F}$ & colon & $\mathrm{T} 2$ & $\mathrm{~N} 2 \mathrm{~b}$ & M1b (lung, peritoneal) \\
\hline 7 & 13 & $\mathrm{~F}$ & colon & $\mathrm{T} 4 \mathrm{~b}$ & NX & M1b (omentum, peritoneal) \\
\hline Serial & AGE (years) & Gender & Site & $\mathrm{T}$ & $\mathrm{N}$ & M \\
\hline 8 & 11 & $\mathrm{~F}$ & colon & $\mathrm{T} 4 \mathrm{a}$ & $\mathrm{N} 2$ & M1b (peritoneal) \\
\hline 9 & 18 & $\mathrm{~F}$ & colon & $\mathrm{T} 2$ & $\mathrm{~N} 2 \mathrm{~b}$ & M1b (bone, omentum) \\
\hline 10 & 9 & M & colon & $\mathrm{T} 3$ & $\mathrm{~N} 2 \mathrm{a}$ & M0 \\
\hline 11 & 15 & $\mathrm{~F}$ & colon & $\mathrm{T} 3$ & $\mathrm{~N} 1 \mathrm{~b}$ & M1b (ovary, hepatic) \\
\hline 12 & 13 & $\mathrm{~F}$ & Rectum & $\mathrm{T} 4 \mathrm{~b}$ & $\mathrm{~N} 2 \mathrm{~b}$ & M0 \\
\hline 13 & 17 & $\mathrm{~F}$ & Rectum & $\mathrm{T} 3$ & $\mathrm{~N} 2 \mathrm{~b}$ & M1a (ovary) \\
\hline 14 & 17 & M & Rectum & $\mathrm{T} 1$ & $\mathrm{~N} 2 \mathrm{~b}$ & M0 \\
\hline 15 & 15 & $\mathrm{~F}$ & Rectum & T3 & $\mathrm{N} 2 \mathrm{~b}$ & M0 \\
\hline
\end{tabular}


Table 1. Continued.

\begin{tabular}{lllllll}
\hline Serial & Stage & Surgery & timing of surgery in relation to Cth & Type of chemotherapy + No. of cycles & $\begin{array}{l}\text { Radiotherapy (Rth) + timing of } \\
\text { Rth }\end{array}$ & status \\
\hline 1 & II A & Yes & UPFRONT & 6 FOLFOX & NOT GIVEN & NOT GIVEN \\
2 & IV A & Yes & UPFRONT & 12 FOLFOX & NOT GIVEN & Died \\
3 & IV b & Yes & UPFRONT & 1 FOLFOX & NOT GIVEN & NOT GIVEN \\
4 & IV b & No & Biopsy only & 3 FOLFOX & NOT GIVEN & Died \\
5 & IV b & No & Biopsy only & FOLFOX & NOT GIVEN & Died \\
6 & IV b & No & Biopsy only & 6 FOLFOX & Died \\
7 & IV b & Yes & Delayed & Type of chemotherapy + No. of cycles & Radiotherapy (Rth) + timing of Rth & Died \\
Serial & Stage & Surgery & timing of surgery in relation to Cth & DOT GIVEN & Died \\
8 & IV b & Yes & UPFRONT & 3 FOLFOX and 7 FOLFIRI & NOT GIVEN & Died \\
9 & IV b & Yes & UPFRONT & 6 FOLFOX and 2 FOLFIRI & NOT GIVEN & Alive \\
10 & III B & Yes & UPFRONT & 6 FOLFOX & NOT GIVEN & Died \\
11 & IV b & Yes & Delayed & 3 FOLFOX and 6 FOLFOXIRI & Yes (preoperative) & Died \\
12 & III C & Yes & Delayed & 6 FOLFOX and 6 FOLFIRI & Yes (Preoperative) & Died \\
13 & IV A & Yes & Delayed & Xeloda and 6 FOLFOX & Yes (Preoperative) & Alive \\
14 & III B & Yes & Delayed & Xeloda, 6 FOLFOX and 6 FOLFOXIRI & Yes (preoperative) & Alive \\
\hline 15 & III C & Yes & Delayed & & & \\
\hline
\end{tabular}

\section{Survival Function}

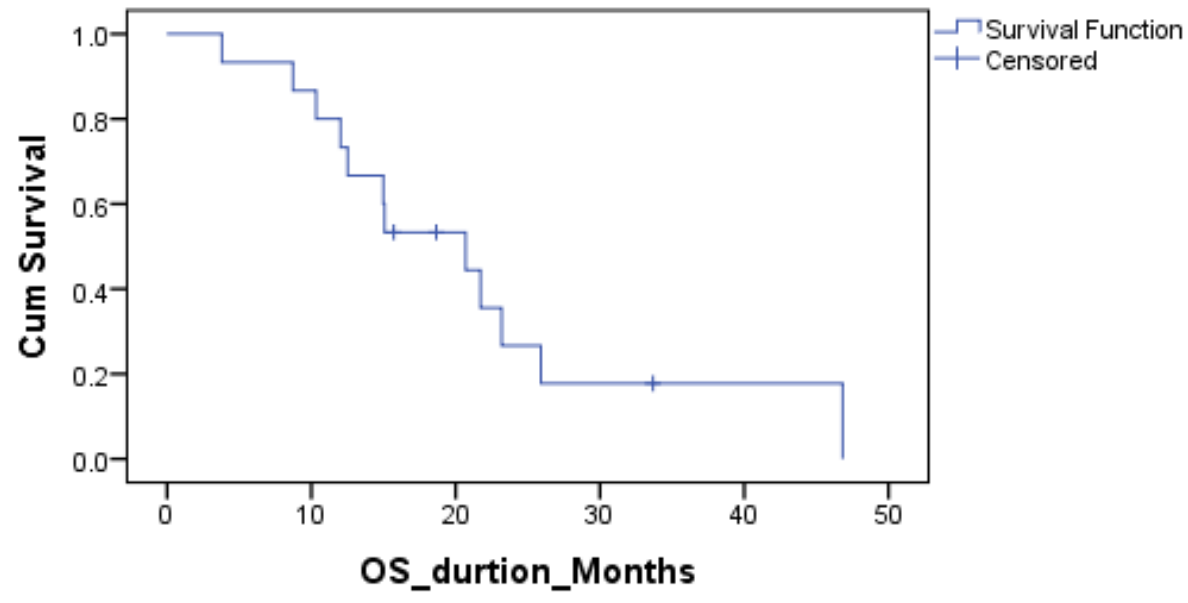

Figure 1. Overall Survival (OS) of the study patients $(n=15)$.

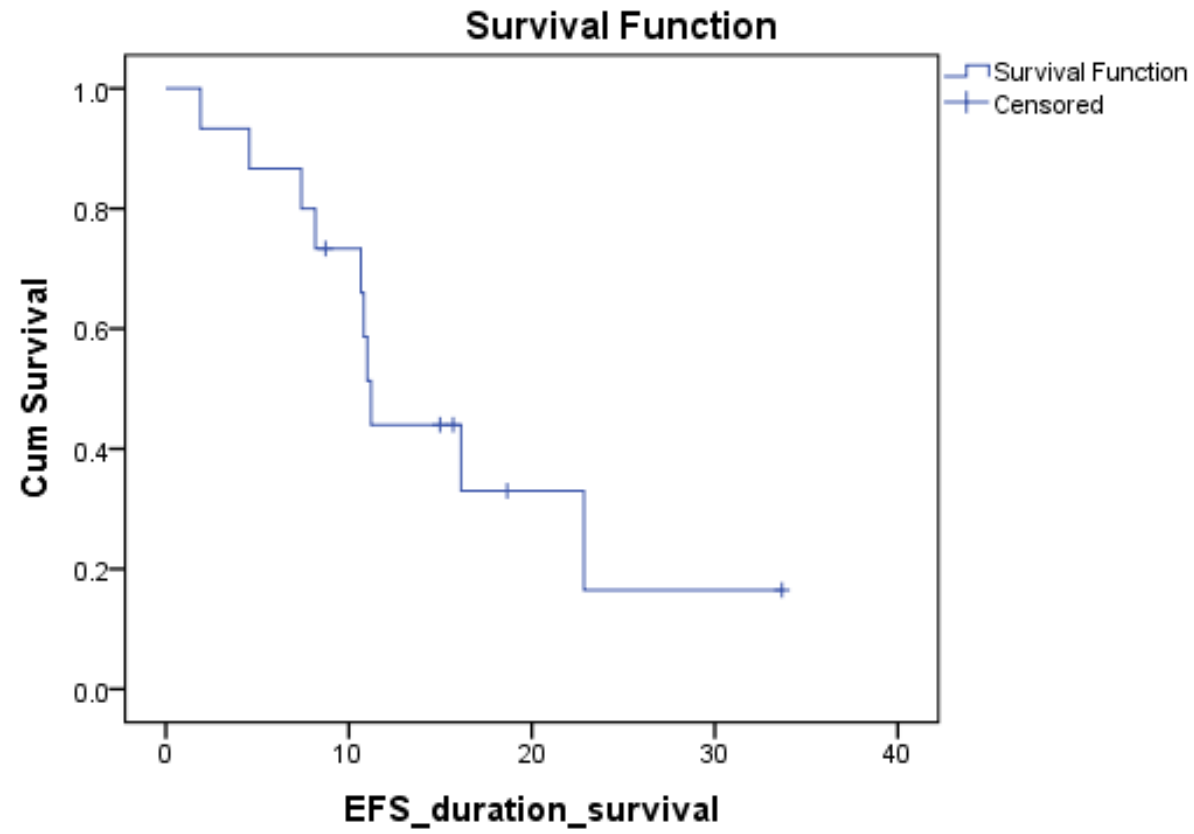

Figure 2. Event free Survival (EFS) of the study patients $(n=1)$. 


\section{Discussion}

Colorectal carcinoma is an extremely rare disease in the pediatric age group with the dismal outcome despite many efforts attempts to improve prognosis [9]. The incidence of CRC is 1 in a million patients under 20 years of age [1]. There is no apparent cause for occurrence of this very aggressive malignant disease in children with much dismal outcome in pediatrics but our opinion, this is mostly due to presence of germ line mutation which predispose these kids to cancer colon and makes this disease very resistant to treatment compared with their adults counterpart and our suggestion may be supported by the evidence that CRC in children is usually not preceded by precancerous conditions as adenomatous polyposis coli unlike adults.

In children with colonic carcinoma, all reports about histopathological types emphasis the predominance of mucinous adenocarcinoma which is found in more than $50 \%$ of patients which is on the contrary different from adults where it is found in only $5-8 \%$ [10]. All patients in our series had unfavorable CRC histopathology types (mucinous adenocarcinoma). HILL et al stated that advanced stage and mucinous histolopathology were negative predictors for poor outcome [11].

Searching for finding the causes of dismal outcome in $\mathrm{CRC}$ in children is that CRC is not on top list of differential diagnosis for patients with abdominal pain, vomiting which results later in an advanced disease at diagnosis and subsequently a poor prognosis. Abdominal pain and vomiting were the most common symptoms in previous reports [12, 13]. In our series abdominal colic was the most common symptoms in 12 cases $(80 \%)$, nausea and vomiting was found in 7 cases $(47 \%)$; these vague symptoms were a major problem which resulted in delayed diagnosis and affecting the prognosis. The duration of symptoms before diagnosis is quite important ranging from 3 to 21 months in different series [14], with a mean of 3.6 months in our study.

Most series reported that $10 \%$ of the childhood colorectal carcinomas have predisposing factors [12]. However, in our series 3 cases $(20 \%)$ seems to have cancer predisposition syndrome; 2 cases had adenomatous familial polyposis and café au lait patches which was reported by the primary physicians as Neurofibromatosis type 1 (NF1) which in our opinion can be more towards CBMMR and also 3rd case had café au lait patches without other clinical stigmata of NF1 and unfortunately we are not applying whole genomic sequencing for proper detection of the underlying predisposing cancer conditions.

Rao et al. reported in 1985 that $67 \%$ of colon carcinoma in children presented as Duke Stage D, 23\% as stage C, and only $10 \%$ as stage B [13]. The staging of our 15 cases according to classification and the American Joint Committee on Cancer (AJCC) Staging System was Stage IIA (1case), III B ( 2 cases), III C ( 2 cases $),$ IV A ( 2 cases $)$ and IV B (8 cases). In our series, 10 cases were metastatic at presentation; one case had rectal carcinoma, while 9 cases were colon carcinoma.

The prognosis in children with carcinoma of the colon is worse than in older patients with a $51 \%$ versus $75 \%$ overall 5 -year survival [13]. In the current study, the 3-year overall survival (OS) was $17.8 \%(\mathrm{SE}+11.1)$ and 3 -year event free Survival (EFS) $16.5 \%$ (SE + 13.5) with median survival 15.7 months (see figures $1 \& 2$ ), additionally Hill et al concluded that 10 years survival was $67 \% \pm 27 \%$ (stage 1 ), $38 \% \pm 15 \%$ (stage 2), $28 \% \pm 11 \%$ (stage III), and $7 \% \pm 4 \%$ (stage 4 ) [11].

Complete surgical resection is the most important prognostic factor for cure in CRC but in pediatrics this is usually difficult due to advanced stage at initial presentation and unresectability of the mass initially making neoadjuvant chemotherapy is referable. The results of many series reported that survival was dependent on the complete initial resection of the primary tumor, the majority of young patients usually present with later stages tumors at diagnosis which makes surgical resectability very difficult [13]. In our series gross total resection was possible in only 8 out of 15 cases either upfront or after neoadjuvant chemo-radiotherapy, however still our results were very dismal which may be related to the presence of distant metastases in the vast majority of cases which even nullifies the impact of achieving gross total resection.

In Children's Cancer Hospital Egypt (CCHE), we established the rare tumors study team in 2014. Since then, we believe that there is no specific treatment modalities for children with CRC different from that used for adult then therapeutic recommendations should be the same as that for adults, i.e. a multimodality treatment. Surgery is the main treatment for localized well defined colorectal cancer and must be proper with radical excision, taking even if it will be associated with extensive resection of nearby tissues.

As for adjuvant therapies, during collection of the data retrospectively we found heterogeneity in the data regarding type of chemotherapy regimen, number of cycles given either neoadjuvant or adjuvant so now in our recently adopted protocol, 5-fluorouracil/folinic acid (5FU/FA) will be our standard as the adjuvant chemotherapy. More controversy exists, on the other hand, concerning the optimal regimen and whether to treat node-negative patients.

Postoperative radiotherapy had a definite role for rectal cancer (particularly combined with 5FU-based chemotherapy), recently been placed on pre-operative radiotherapy (PRT) a collection of number of trials proved that preoperative radiotherapy probably reduces overall mortality at 4 to 12 years and for every 1000 people who undergo surgery alone, 454 would die compared with 45 fewer in the preoperative radiotherapy group. However, in trials using total meso rectal excision (TME) there was a proof that there is no or little effect of the PRT on survival [15], but all this data is projected from adult series which is different from the pediatric series

Hill et al stated that advanced stage and mucinous histolopathology were significant predictors of poor outcome. Stage-specific survival at 10 years was $67 \% \pm 27 \%$ 
(stage 1), 38\% $\pm 15 \%$ (stage 2), $28 \% \pm 11 \%$ (stage III), and $7 \% \pm 4 \%$ (stage 4 ) [11].

In summary, awareness that $\mathrm{CRC}$ does occur in pediatric age in context of presence of recurrent abdominal pain, cachexia, loosing of weight and bleeding per rectum, then pediatricians had to refer suspected cases for a specialized center which will be more experienced in management of CRC patients. Close cooperation with adult surgeons and oncologists is crucial to improve quality of cure for children and adolescents with CRC. In Children's Cancer Hospital Egypt, we started to adopt recently a fixed protocol for colorectal carcinoma management in pediatric age group and supports the feasibility of international studies as we are the biggest center in Egypt, treating majority of cancer patients all over Egypt and nearby countries.

\section{Conclusions}

Clinical presentation of CRC in pediatrics is similar as in adults with a higher incidence of delayed diagnosis and advanced stages which may contribute to poor outcome in childhood CRC due to the absence of familiarity to CRC in the differential diagnosis of abdominal pain and disturbed bowel habits. The high frequency of mucinous adenocarcinoma in our study may explain the poor outcome of our patients. There is no known protocols for pediatric age with $\mathrm{CRC}$, then following adult protocols for treatment. Surgery remains the mainstay of treatment as in adults. Preoperative radiotherapy for rectal adenocarcinoma, offers better prognosis. Chemotherapy plays a role in the metastatic disease and can downstage the primary tumor for better local control. Searching for cancer predisposition syndrome or presence of germ line mutation which predispose these kids to cancer colon and makes this disease very resistant to treatment compared to adult's results is very important.

\section{Acknowledgements}

An abstract of this paper was presented at the 50th Congress of the International Society of Pediatric Oncology (SIOP) in Kyoto Japan as a poster with number PO-649.

\section{References}

[1] Torre LA, Bray F, Siegel RL, Ferlay J, Lortet-Tieulent J, Jemal A. (2006). Global cancer statistics, 2012. CA Cancer J Clin. 2015; 65: 87-108.
[2] Huxley RR, Ansary-Moghaddam A, Clifton P, Czernichow S, Parr CL, Woodward M.(2009). The impact of dietary and lifestyle risk factors on risk of colorectal cancer: a quantitative overview of the epidemiological evidence. Int J Cancer. 2009; 125: $171-180$.

[3] Jemal A, Center MM, DeSantis C, Ward EM. (2010). Global patterns of cancer incidence andmortality rates and trends. Cancer Epidemiol Biomarkers Prev. 2010; 19: 1893-1907.

[4] Spunt S, Furman W, La Quaglia M, et al, (2007). Colorectal Carcinoma in Childhood and Adolescence, Bethesda, MD, National Cancer Institute, NIH Pub. No. 06-5767, pp. 123, 2006-134.

[5] Turnbull RB Jr, Kyle K, Watson FR, et al. (1967) Cancer of the colon: The influence of the no-touch isolation technique on survival rates. Ann Surg 1967; 166: 420-427.

[6] AJCC Cance Staging Manual. 6th ed. New York: SpringerVerlag; 2002.

[7] Ferrari A, Bertario L, Signoroni S, et al (2008). Colorectal Carcinoma in Children and Adolescents, onlinelibrary.wiley.com/doi/10.1002/pbc.21220.

[8] Kaplan E, Meier P. (1958). Non-parametric estimation from incomplete observation. J Am Stat Assoc 1958; 53: 457-481.

[9] Khan AM, Doig CM, Dickson AP. (1997). Advanced colonic carcinoma in children. Postgrad Med J. 1997; 73: 169-170.

[10] Narla LD, Hingsbergen EA, Jones JE. (1999). Adult diseases in children. Pediatr Radiol. 1999; 29: 244-254.

[11] Hill DA, Furman WL, Billups CA, Riedley SE, Cain AM, Rao BN, Pratt CB, Spunt SL.(2007). Colorectal carcinoma in childhood and adolescence: a clinicopathologic review J Clin Oncol. 2007 Dec 20; 25 (36): 5808-14.

[12] Karnak I, Ciftci AO, Senocak ME, Büyu"kpamukc,u N (1999) Colorectal carcinoma in children. J Pediatr Surg 34: 1499-1504.

[13] Rao BN, Pratt CB, Fleming ID et al (1985) Colon carcinoma in children and adolescents: a review of 30 cases. Cancer 55: $1322-1326$

[14] Brown RA, Rode H, Millar AJW et al (1992) Colorectal carcinoma in children. J Pediatr Surg 27: 919-921.

[15] Abraha I, Aristei C, Palumbo I, Lupattelli M, Trastulli S, Cirocchi R, De Florio R, Valentini V. (2018). Preoperative radiotherapy and curative surgery for the management of localised rectal carcinoma. Cochrane Database of Systematic Reviews 2018, Issue 10. Art. No.: CD002102. DOI: $10.1002 / 14651858$. 